\title{
Trigger Factor Forms a Protective Shield for Nascent Polypeptides at the Ribosome*
}

Received for publication, November 17, 2005, and in revised form, January 4, 2006 Published, JBC Papers in Press, January 5, 2006, DOI 10.1074/jbc.M512345200

\author{
Anja Hoffmann, Frieder Merz, Anna Rutkowska, Beate Zachmann-Brand, Elke Deuerling ${ }^{1}$, and Bernd Bukau ${ }^{2}$ \\ From the Zentrum für Molekulare Biologie Heidelberg (ZMBH), Im Neuenheimer Feld 282, Universität Heidelberg, \\ 69120 Heidelberg, Germany
}

In prokaryotes, the ribosome-associated Trigger Factor is the first chaperone newly synthesized polypeptides encounter when they emerge from the ribosomal exit tunnel. The effects that Trigger Factor exerts on nascent polypeptides, however, remain unclear. Here we analyzed the potential of the Trigger Factor to shield nascent polypeptides at the ribosome. A set of arrested nascent polypeptides differing in origin, size, and folding status were synthesized in an Escherichia coli-based in vitro transcription/translation system and tested for sensitivity to degradation by the unspecific protease proteinase $\mathrm{K}$. In the absence of Trigger Factor, nascent polypeptides exposed outside the ribosomal exit tunnel were rapidly degraded unless they were folded into a compact domain. The presence of Trigger Factor, as well as a Trigger Factor fragment lacking its peptidyl-prolyl isomerase domain, counteracted degradation of all unfolded nascent polypeptides tested. This protective function was specific for ribosome-tethered Trigger Factor, since neither non-ribosomal Trigger Factor nor the DnaK system, which cooperates with Trigger Factor in the folding process in vivo, revealed a comparable efficiency in protection. Furthermore, shielding by Trigger Factor was not restricted to short stretches of nascent chains but was evident for large, non-native nascent polypeptides exposing up to $41 \mathrm{kDa}$ outside the ribosome. We suggest that Trigger Factor supports productive de novo folding by shielding nascent polypeptides on the ribosome thereby preventing untimely degradation or aggregation processes. This protected environment provided by Trigger Factor might be particularly important for large multidomain proteins to fold productively into their native states.

Cells employ a large arsenal of molecular chaperones to promote the folding of newly synthesized proteins in the cytosol (1-3). At the forefront are chaperones, which transiently bind to ribosomes and thereby are optimally positioned to associate with emerging nascent polypeptides. In eubacteria, Trigger Factor (TF) ${ }^{3}$ binds to ribosomes near the exit of the peptide tunnel through major contacts to L23 (4-7) and

* This work was supported by grants from the Deutsche Forschungsgemeinschaft (DFG) (to B. B. and E. D.) and the Human Frontier Science Program (to E. D), a Heisenberg Fellowship from the DFG (to E. D.), a fellowship from the Boehringer Ingelheim Fonds (to A. H.), and by a grant from the Fonds der Chemischen Industrie (to B. B.). The costs of publication of this article were defrayed in part by the payment of page charges. This article must therefore be hereby marked "advertisement" in accordance with 18 U.S.C. Section 1734 solely to indicate this fact.

${ }^{1}$ To whom correspondence may be addressed. Tel.: 49-6221-546870; Fax: 49-6221545894; E-mail: e.deuerling@zmbh.uni-heidelberg.de.

${ }^{2}$ To whom correspondence may be addressed. Tel.: 49-6221-546870; Fax: 49-6221545894; E-mail: bukau@zmbh.uni-heidelberg.de.

${ }^{3}$ The abbreviations used are: TF, Trigger Factor; PPlase, peptidyl-prolyl cis/trans-isomerase; aa, amino acid(s); t/t, transcription/translation; ICDH, isocitrate dehydrogenase; wt, wild type; KJE, DnaK-DnaJ-GrpE; NC, Trigger Factor fragment consisting of the Nand C-terminal domains; SH3, Src homology 3; BSA, bovine serum albumin; PK, proteinase $\mathrm{K}$. interacts co-translationally with nascent polypeptides $(8,9)$. Downstream of TF, the DnaK-DnaJ-GrpE (referred to as KJE) and GroELGroES chaperone systems assist further folding steps to the native state $(2,10)$. Together these chaperones form a folding network of considerable robustness. Thus, the lack of TF can be compensated by activity of the KJE system, while simultaneous deletion of $d n a K$ and the TF encoding tig gene results in synthetic lethality at $\geq 30{ }^{\circ} \mathrm{C}$ and massive protein aggregation (11-13).

TF is a three-domain protein of $48 \mathrm{kDa}$ that adopts an unusual extended conformation (Fig. $1 A)(5,14)$. The N-terminal domain builds the "tail" of the molecule and harbors the "TF-signature motif," which mediates ribosome docking (4). A peptidyl-prolyl cis/trans isomerase (PPIase) domain with structural homology to FK506-binding proteins $(9,16)$ is located at the other end of the molecule, forming the "head" of TF. The C-terminal domain of TF resides between the head and tail domains to build "back" and "arms" of TF. Recently, three different crystal structures of $50 \mathrm{~S}$ ribosomes in complex with the N-terminal ribosome-binding domain of TF were solved allowing for the modeling of the entire chaperone bound to the large ribosomal subunit (5-7). On one hand, these structural models consistently suggest that TF hunches over the ribosomal exit site (Fig. $1 B$ ) thereby orienting its hydrophobic inner surface toward emerging nascent polypeptides. On the other hand, the models predict different volumes for the space formed by the ribosomal surface and the $\mathrm{N}$-terminal tail and $\mathrm{C}$-terminal arms of TF. While two models propose that this space resembles a cavity which is large enough to accommodate protein domains or small proteins of $\sim 14 \mathrm{kDa}$ or even larger $(5,6)$, the third model suggests a rather narrow crevice more suitable to bind an unfolded polypeptide (7). These contradictory models of the conformation of TF on the ribosome make it difficult to define a mechanism of action for TF and do not further clarify what effects TF exerts on nascent polypeptides.

However, they imply that part of the intrinsic chaperone function of $\mathrm{TF}$ at the ribosome is to form a protective shield restricting the accessibility of nascent chains to the crowded cellular environment. Here we assign this basic shielding function to TF. Applying protease protection assays in an in vitro transcription/translation system we (i) probed for the proteolytic sensitivity of nascent polypeptides, (ii) examined the potential of TF to provide a protected environment for diverse nascent polypeptides differing in size and folding status, and (iii) analyzed whether the cooperating KJE chaperone system acting downstream of TF can substitute for TF in protecting nascent polypeptides from proteolytic degradation.

\section{EXPERIMENTAL PROCEDURES}

Cloning of Src Homology 3 (SH3) and m10 Constructs-To arrest SH3 and $\mathrm{m} 10$ domains at the ribosome, $\mathrm{SH} 3$ and m10 coding sequences lacking stop codons were fused at their $3^{\prime}$ ends to a nucleotide sequence encoding a 41-aa linker that contained a flexible serine/glycine-rich region (GSGGGSG) and the SecM arrest sequence. SH3 and m10 


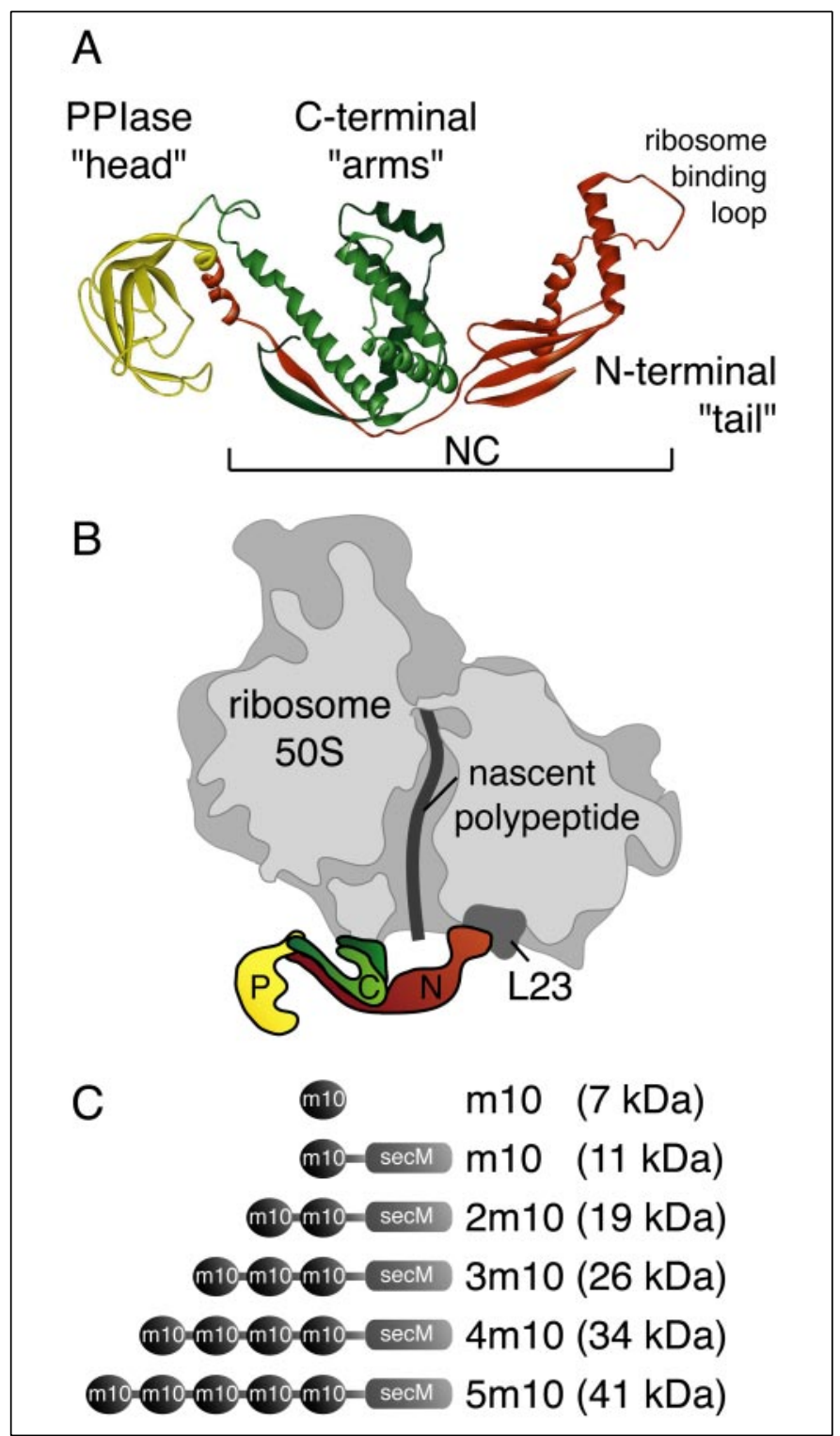

FIGURE 1. TF and model substrates. Displayed are the crystal structure (Protein Data Bank code 1W26) of soluble TF $(A)$ and schematic representations of ribosome-bound TF $(B)$ and of the m10 constructs used as model substrates in this study $(C)$. The N-terminal domain of TF ("N") is depicted in red, the PPlase domain (" $P$ ") in yellow and the C-terminal $\left(" C{ }^{\prime \prime}\right)$ domain in green $(A$ and $B)$. The NC fragment of TF lacking the PPlase is indicated $(A)$.

domains were amplified from pBAT4 plasmids (17) by PCR using the primer NcoI-f (5' -GATATATCCATGGTTGATGAAACTGGAAAAGAGC-3'), which introduced an additional gtt codon following the start codon, and the primer BamHI-r (5' -GCCAGAGCCACCGCCGGATCCATCTAGTTTTTTCACATAGGC-3'). The linker was amplified using pUC19StrepFtsQSecM (18) as template and primers BamHI-f (5' GGATCCGGCGGTGGCTCTGGCTCTGAAAAGGGTTATCGCATTG-3') and HindIII-r (5'-CGCCAAGCTTATCAATGCATCATCATCATC-3'). Resultant PCR products were utilized in two different fusion PCR reactions (either SH3 and linker or $\mathrm{m} 10$ and linker) with NcoI-f and HindIII-r as primers. Fusion products were digested with NcoI and HindIII and ligated into pBAT4, which had been cut with NcoI and HindIII to excise the SH3 insert, giving rise to the plasmids pBAT4SH3-SecM and pBAT4-m10-SecM.

Plasmid pBAT4-m10-SecM was used to construct vectors coding for two to five m10 domains (with individual m10 domains separated by either a short GASS or a GSGS linker dependent on the restriction site introduced for cloning). Plasmid pBAT4-m10-SecM served as template in two different PCR reactions amplifying the m10 sequence (primers BamHI-f2 (5' -GCGGATCCGGCTCTATGGTTGATGAAACTGG-3') and SacII-r (5'-GCCACCGCCCGCGGAATCTAGTTTTTTCAC-3')) or the SecM linker (primers SacII-f (5'-CTGGGTTCCGCGGGCGGTGGCTCTGGCTCTG-3') and HindIII-r). PCR products were digested with BamHI and SacII or SacII and HindIII, respectively, and ligated together into pBAT4-m10-SecM digested with BamHI and HindIII resulting in pBAT4-2m10-SecM encoding for an m10 dimer.

Similarly, plasmid pBAT4-m10-SecM was used as template to amplify the m10 sequence using the primers BamHI-f2 and XhoI-r (5' -CGCTCGAGGCTCCATCTAGTTTTTTCAC-3') or the primers XhoI-f (5'-GCCTCGAGCATGGTTGATGAAACTGG-3') and SacII-r. PCR products were digested with BamHI and XhoI or XhoI and SacII, respectively, and both products were ligated into pBAT4-2m10SecM digested with BamHI and SacII giving rise to the pBAT4-3m10SecM plasmid containing three consecutive m10 sequences.

To generate plasmid pBAT4-4m10-SecM, a sequence encoding two m10 domains was amplified using pBAT4-2m10-SecM as template and XhoI-f and SacII-r as primers. The amplified product was digested with XhoI and SacII and ligated into the pBAT4-3m10-SecM vector digested with XhoI and SacII.

The final plasmid pBAT4-5m10-SecM encoding for a stalled m10 pentamer was constructed by cloning an additional m10 domain (amplified by PCR from pBAT4-m10-SecM with primers XhoI-f and XhoI-r and digested with XhoI) into the pBAT4-4m10-SecM plasmid linearized with XhoI. A scheme of the resultant $\mathrm{m} 10$ constructs that can be generated from the depicted plasmids is shown in Fig. 1C.

Purification of Chaperones-C-terminally His-tagged TF, the NC fragment (19), and mutant TF-FRK/AAA (4) were expressed from pDS56 plasmids (20) in MC4100 $\Delta$ tig strains (11) and purified as described (19). DnaK and DnaJ were purified according to published protocols $(21,22)$. GrpE carrying a C-terminally fused $\mathrm{His}_{6}$-tag was expressed from plasmid pUHE in an MC4100 $\Delta d n a K$ strain (23) and purified via nickel-nitrilotriacetic acid-agarose and anion exchange chromatography (ResourceQ6 column, Amersham Biosciences).

In Vitro Transcription/Translation ( $t / t$ ) System-Translation extracts were prepared from an MC4100 $\Delta$ tig (11) strain according to published protocols (24) and synthesis of arrested isocitrate dehydrogenase (ICDH) chains was performed as described (12). Truncated mRNAs encoding the ICDH constructs of $11,19,32,39$, and $45 \mathrm{kDa}$ were generated by supplementing the $\mathrm{t} / \mathrm{t}$ system with $\mathrm{RNaseH}$ and antisense oligos 5' -CCGGAGTGGTCAGCGGACC-3' ', 5' -CCCCCATCTCTTCACGCAGG-3', 5' -GGCGATAACATCATATTCAGCCGG3', 5' -AGCGGAGAGAATAATAGAGCC-3', and 5' -TTACATGTTTTCGATGATCGC-3', respectively. SH3 and m10 constructs were expressed from the plasmids depicted above. Wild type (wt) and mutant L23 VSE/AAA ribosomes were purified as described below and added to the $t / t$ system in a final concentration of $400 \mathrm{nM}$.

Ribosome Purification-wt ribosomes were purified from strain MC4100 $\Delta$ tig (11), mutant VSE/AAA ribosomes from strain MC4100 $\Delta t i g \Delta r p l W$ expressing L23 VSE/AAA mutant protein upon isopropyl $\beta$-D-thiogalactopyranoside induction (4). Strains were grown at $37^{\circ} \mathrm{C}$ to an $A_{600 \mathrm{~nm}}$ of 1.0 in LB medium (wt) or LB medium supplemented with $250 \mu \mathrm{M}$ isopropyl $\beta$-D-thiogalactopyranoside (VSE/AAA mutant), harvested by centrifugation, and resuspended in $1.5 \mathrm{ml} / \mathrm{g}$ wet pellet $\mathrm{S} 30$ buffer (50 mM triethanolamine ( $\mathrm{pH} 8.0$ ), $50 \mathrm{~mm}$ KOAc, $15 \mathrm{~mm}$ $\mathrm{Mg}(\mathrm{OAc})_{2}, 1 \mathrm{~mm}$ dithiothreitol) supplemented with $1 \mathrm{~mm}$ phenylmeth- 
ylsulfonyl fluoride. After cell lysis by French press and removal of cell debris by centrifugation ( 2 times $30,000 \times g$, $30 \mathrm{~min}, 4{ }^{\circ} \mathrm{C}$ ), cleared supernatants (concentration of KOAc adjusted to $1 \mathrm{M}$ in a final volume of $15 \mathrm{ml}$ ) were loaded onto 45-ml high salt sucrose cushions (25\% sucrose, $50 \mathrm{~mm}$ triethanolamine ( $\mathrm{pH}$ 8.0), 1 м KOAc, $6 \mathrm{~mm} \mathrm{Mg}(\mathrm{OAc})_{2}$, 4 mм $\beta$-mercaptoethanol). Ribosomes were pelleted by centrifugation $\left(44,000 \mathrm{rpm}, 4 \mathrm{~h}, 4{ }^{\circ} \mathrm{C}\right)$ in a Ti45 rotor (Beckman), rinsed with $\mathrm{S} 30$ buffer and resuspended overnight on ice in S30 buffer. The high salt sucrose cushion centrifugation was repeated, and pelleted ribosomes were resuspended overnight on ice in storage buffer ( $50 \mathrm{~mm}$ triethanolamine (pH 8.0), $50 \mathrm{~mm} \mathrm{KOAc,} 6 \mathrm{~mm} \mathrm{Mg(OAc})_{2}, 4 \mathrm{~mm} \beta$-mercaptoethanol), aliquotted, frozen in liquid nitrogen, and stored at $-80^{\circ} \mathrm{C}$.

Proteinase $K$ Digests-Synthesis of $\left[{ }^{35} \mathrm{~S}\right]$ Methionine-labeled constructs in the in vitro $\mathrm{t} / \mathrm{t}$ system was performed in the presence of chaperones or bovine serum albumin (BSA) added in concentrations of $5 \mu \mathrm{M}$ (BSA, TF, NC, TF-FRK/AAA, DnaK) and $1 \mu \mathrm{M}$ (DnaJ, GrpE). 15-20 min after initiation, translation was stopped with $2 \mathrm{~mm}$ chloramphenicol (arrested polypeptides) or $2 \mathrm{~mm}$ puromycin (released polypeptides), and subsequently proteinase $\mathrm{K}$ (PK) was added in a final concentration of 5 $\mu \mathrm{g} / \mathrm{ml}$. In the experiment depicted in Fig. $5 C$, BSA and TF were not present during synthesis but added posttranslationally 5 min before PK. In co-translational PK digests, arrested ICDH or m10 dimer (2m10) were synthesized in the presence of $1 \mu \mathrm{M}$ TF, TF-FRK/AAA, or BSA, and $\mathrm{PK}$ was added during ongoing translation $(8 \mathrm{~min}(\mathrm{ICDH})$ or $5 \mathrm{~min}$ (2m10) after initiation of expression) in final concentrations of 0.7 $\mu \mathrm{g} / \mathrm{ml}(\mathrm{ICDH})$ or $1 \mu \mathrm{g} / \mathrm{ml}(2 \mathrm{~m} 10)$. Posttranslational digests were carried out on ice, co-translational digests at $37^{\circ} \mathrm{C}$. Before $(0 \mathrm{~min}), 2 \mathrm{~min}$, and $4 \mathrm{~min}$ after the addition of $\mathrm{PK}$, aliquots were taken, treated with 2 $\mathrm{mM}$ phenylmethylsulfonyl fluoride to inhibit $\mathrm{PK}$, and loaded onto sucrose cushions (20\% sucrose, 50 mm HEPES-KOH (pH 7.5), $100 \mathrm{~mm}$ KOAc, $10 \mathrm{~mm} \mathrm{Mg}(\mathrm{OAc})_{2}$, $4 \mathrm{~mm} \beta$-mercaptoethanol, $2 \mathrm{~mm}$ chloramphenicol, $2 \mathrm{~mm}$ phenylmethylsulfonyl fluoride; 2.5 volumes cushion per 1 volume of sample). Ribosome-nascent chain complexes were pelleted by centrifugation in a TLA-100 rotor (Beckman) at 74,000 rpm for 70 min at $4{ }^{\circ} \mathrm{C}$. Ribosomal pellets (arrested constructs) or supernatants (released constructs) were separated on Tricine gels, and ${ }^{35} \mathrm{~S}$-labeled polypeptides were visualized by autoradiography.

\section{RESULTS}

Trigger Factor Shields Nascent Polypeptides from Degradation-We investigated the potential of TF to shield nascent polypeptide chains by protease protection experiments in an in vitro transcription/translation system ( $\mathrm{t} / \mathrm{t}$ system) derived from Escherichia coli $\Delta$ tig cells, which allows the controlled co- or posttranslational addition of TF and its mutant variants. $\left[{ }^{35} \mathrm{~S}\right]$ Methionine-radiolabeled nascent chains derived from different model proteins were generated. One model protein was E. coli ICDH (417 aa, $46 \mathrm{kDa}$ ), a previously identified in vivo substrate of TF (12), of which we synthesized a truncated construct (177 aa, $19 \mathrm{kDa}$ ). The conformation of this truncated ICDH version cannot be deduced from the known atomic structure of full-length ICDH (25). To investigate substrates with defined structures, we generated nascent polypeptides derived from the SH3 domain of $\alpha$-spectrin and its mutant variant m10. While wt SH3 folds into a small (62 aa, $7 \mathrm{kDa}$ ) globular $\beta$-sheet structure (26), m10 harbors two point mutations (W41G and W42V) that prevent native folding and instead generate a random coil structure (17). To produce arrested $\mathrm{SH} 3$ and $\mathrm{m} 10$ polypeptides exposing the entire SH3 or m10 domains outside the ribosomal exit tunnel, we introduced a C-terminal 41-aa linker. This linker carried the SecM stalling sequence known to arrest translation and was long enough to extend through the entire length of the ribosomal peptide tunnel $(27,28)$.
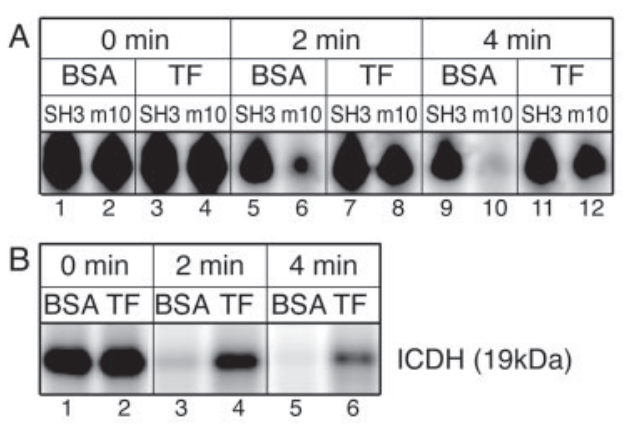

FIGURE 2. TF shields nascent polypeptides from degradation by proteinase $\mathrm{K}$. $\left.{ }^{35} \mathrm{~S}\right]$ Methionine-labeled arrested nascent polypeptides were synthesized in an E. coli in vitro $\mathrm{t} / \mathrm{t}$ system in the presence of $5 \mu \mathrm{M}$ TF or $5 \mu \mathrm{M}$ BSA as control. After synthesis, PK ( 5 $\mathrm{ng} / \mu \mathrm{l})$ was added and at indicated time points of PK digest $(0,2$, and $4 \mathrm{~min})$ degradation was stopped, and the remaining ribosome-nascent chain complexes were isolated by sucrose cushion centrifugation. Subsequently, polypeptides were separated on Tricine gels and visualized by autoradiography. Full-length products of arrested $\mathrm{SH} 3$ and $\mathrm{m} 10$ are shown in $A$; nascent ICDH polypeptides are depicted in $B$.

To probe for degradation of nascent chains, the model substrates were synthesized in the presence of TF or an equal concentration of BSA as control and the unspecific protease proteinase $\mathrm{K}$ was added after translation. In the absence of $\mathrm{TF}$, the arrested $\mathrm{SH} 3$ domain showed significant resistance to proteinase $\mathrm{K}$ indicating that ribosome-tethered $\mathrm{SH} 3$ reaches a protease-resistant, folded state (Fig. $2 A$, lanes 5 and 9). In contrast, proteinase $\mathrm{K}$ treatment resulted in almost complete degradation of the arrested chains of 10 (Fig. $2 A$, lanes 6 and 10) and ICDH (Fig. 2B, lanes 3 and 5) in the absence of TF, which suggests that truncated ICDH is not compactly folded. Thus our experimental setup allows to discriminate between folded and unfolded states of ribosomeassociated nascent chains.

The presence of TF, which was added prior to the start of protein synthesis in a concentration $(5 \mu \mathrm{M})$ sufficient to saturate ribosomes (400 $\mathrm{nM}$ ), strongly reduced degradation of $\mathrm{m} 10$ and ICDH nascent chains by proteinase K (Fig. 2A, lanes 8 and 12; Fig. $2 B$, lanes 4 and 6). This protection was not due to competition between TF and nascent chains for degradation by proteinase $\mathrm{K}$, since (i) addition of BSA in the control reaction did not prevent degradation of nascent chains (Fig. $2 A$, lanes 6 and 10; Fig. 2B, lanes 3 and 5), and (ii) TF remained stable during proteinase $\mathrm{K}$ treatment (data not shown). Protection by TF was not dependent on the type of protease employed in our system, since TF also shielded ICDH $(19 \mathrm{kDa})$ and $\mathrm{m} 10$ from proteolytic attack by trypsin (data not shown).

These results show that nascent chains exposed outside the ribosomal tunnel are highly sensitive to proteolysis unless they have a compact fold. Furthermore, they demonstrate that TF is capable of restricting the accessibility of non-native $\mathrm{m} 10(11 \mathrm{kDa})$ and ICDH $(19 \mathrm{kDa})$ constructs. Assuming that 35 aa $(4 \mathrm{kDa})$ of nascent chains are buried inside the ribosomal tunnel, TF protected polypeptides of 7 and $15 \mathrm{kDa}$, respectively.

Ribosome Attachment Is Prerequisite for the Shielding Function of TF-To determine whether the protective activity of TF requires its association with the ribosome, we employed a t/t system with ribosomes mutated in the L23-docking site of TF (L23-VSE/AAA) (4) and added the TF-FRK/AAA mutant protein, which is selectively defective in ribosome association (4). Under these conditions the protective effect of TF was abolished for the arrested $\mathrm{m} 10$ and ICDH polypeptides as both constructs were efficiently degraded by proteinase $\mathrm{K}$ in the presence and absence of TF-FRK/AAA (Fig. 3A). Furthermore, we tested whether TF protects newly synthesized proteins after their release from the ribosome. When non-arrested ICDH $(19 \mathrm{kDa})$ and m10 domain $(7 \mathrm{kDa}$, 
lacking the linker with the SecM-arrest sequence) were produced in the presence of wt ribosomes and wt TF, TF failed to prevent the degradation of these soluble polypeptides by proteinase K (Fig. 3B). We conclude that TF displayed its shielding effect solely for nascent chains still attached to ribosomes and that ribosome binding of TF was prerequisite for this protective function.

Trigger Factor Protects Large Nascent Polypeptides-We evaluated the size limits of the nascent polypeptides, which can be protected by TF. Of interest was to compare the functionality of full-length TF with a $\mathrm{TF}$ fragment (NC, Fig. 1A) that lacks the PPIase domain, which is located distant to the ribosomal peptide tunnel exit $(5,19)$. To generate

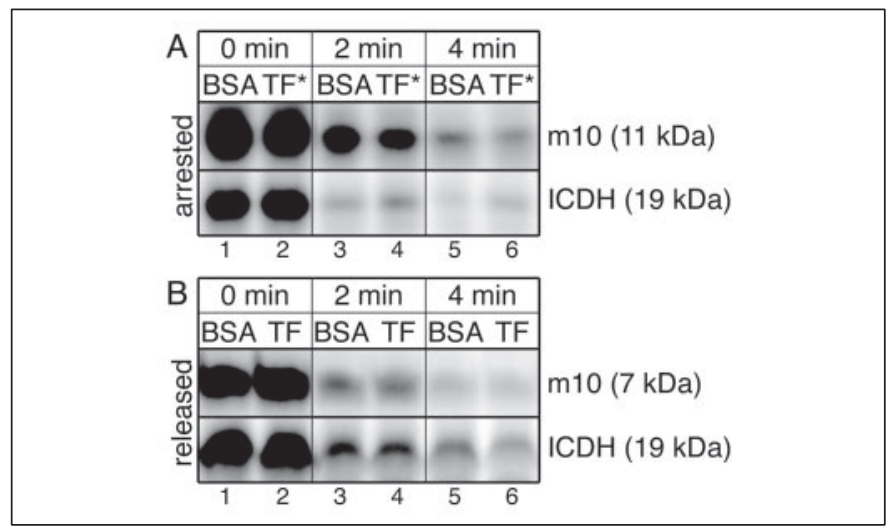

FIGURE 3. Ribosome attachment is prerequisite for the protective function of TF. $A$ arrested ICDH and $\mathrm{m} 10$ polypeptides were synthesized in the presence of the TF-FRK/ AAA (TF*) ribosome-binding mutant $(5 \mu \mathrm{M})$ or BSA $(5 \mu \mathrm{M})$ using $\mathrm{L} 23$ mutant ribosomes (VSE/AAA). $B$, puromycin-released ICDH and m10 (lacking the arrest-mediating linker) were translated by wt ribosomes in the presence of $5 \mu \mathrm{m}$ wt TF or $5 \mu \mathrm{M}$ BSA. All samples were treated as described in the legend to Fig. 2. nascent chains of varying length but unaltered folding status, we constructed genes encoding an m10 dimer $(2 \mathrm{~m} 10,19 \mathrm{kDa})$, trimer $(3 \mathrm{~m} 10$, $26 \mathrm{kDa})$, tetramer $(4 \mathrm{~m} 10,34 \mathrm{kDa})$, or pentamer $(5 \mathrm{~m} 10,41 \mathrm{kDa})$, each with the C-terminal SecM-arrest sequence and short linkers connecting the $\mathrm{m} 10$ domains (Fig. $1 C$ ). The $\mathrm{m} 10$ variants were synthesized in the $t / t$ system in the presence of BSA, TF, or the NC fragment of TF lacking the PPIase domain. Upon subsequent addition of proteinase $\mathrm{K}$ all $\mathrm{m} 10$ constructs synthesized in the presence of BSA were highly susceptible to degradation revealing faster degradation kinetics with increasing size (Fig. 4A, compare for example lanes 16, 19, 22, 25, and 28). The presence of TF or NC retarded proteolysis of all m10 constructs tested. While TF and the $\mathrm{NC}$ fragment similarly left the majority of monomeric and dimeric $\mathrm{m} 10$ undigested after $2 \mathrm{~min}$ of proteinase $\mathrm{K}$ treatment (Fig. $4 \mathrm{~A}$, lanes $17,18,20$, and 21), full-length TF was slightly more efficient in protecting larger nascent chains exposing more than $15 \mathrm{kDa}$ outside the ribosomal tunnel (Fig. 4A, compare for example lanes 23 and 24).

In similar experiments with arrested ICDH polypeptides of 19, 32, 39, and $45 \mathrm{kDa}$, TF and $\mathrm{NC}$ shielded all tested polypeptides from degradation by proteinase $\mathrm{K}$ as compared with the BSA control (Fig. $4 B$ ). In the case of the ICDH constructs, degradation kinetics did not correlate with size suggesting different folds in the different truncations (Fig. 4B, compare lanes $13,16,19$, and 22). In this set of experiments, a contribution of the PPIase to the shielding activity of TF was detectable for the 32and $39-\mathrm{kDa}$ truncations but not for the 19 - and $45-\mathrm{kDa}$ constructs (Fig. $4 B$, e.g. compare lanes 17 and 18 and 20 and 21 with lanes 14 and 15 and 23 and 24 ).

These findings show that $\mathrm{NC}$ provides the major contribution to the shielding activity of TF, which is evident for large non-native nascent polypeptides exposing up to $41 \mathrm{kDa}$ outside the ribosomal tunnel.
FIGURE 4. TF shields large nascent polypeptides from proteolytic attack. Arrested nascent polypeptides of different length derived from $\mathrm{m} 10(A)$ or $\mathrm{ICDH}(B)$ were synthesized in the presence of BSA, full-length TF, or a TF fragment lacking the PPlase domain (NC; see Fig. $1 A$ ) and subjected to PK digest as outlined in the legend to Fig. 2. Boxed areas highlight the residual full-length nascent chains after 2 min of PK digest. Please note that smaller byproducts including a prominent 6-kDa product for various ICDH chains (indicated by an asterisk) appear during synthesis for unknown reasons. Bands running 30 $\mathrm{kDa}$ higher than the individual full-length products represent full-length polypeptides attached to tRNA.

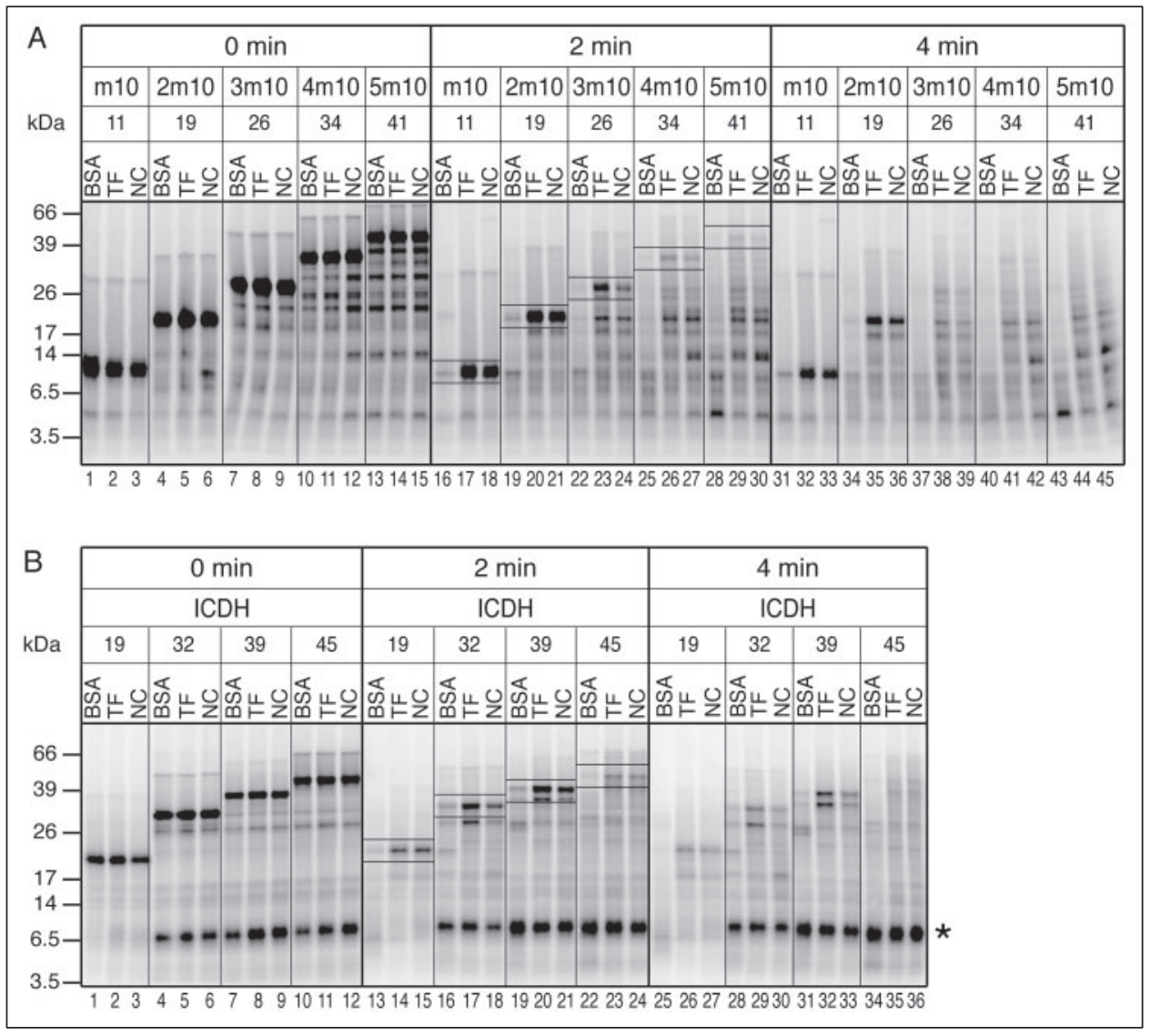


TF Is Able to Protect Arrested Nascent Chains Co- and Posttranslationally - In all experiments described above, proteinase $\mathrm{K}$ was added after translation. To confirm that TF shields nascent polypeptides during ongoing translation, we added proteinase $\mathrm{K}$ to our $\mathrm{t} / \mathrm{t}$ system immediately after initiation of translation. In the absence of $\mathrm{TF}$, proteinase $\mathrm{K}$ addition resulted in the rapid degradation of nascent m10 dimer and 19-kDa ICDH construct, since no accumulation of these products was observed in contrast to the control reactions lacking proteinase K (Fig. 5, $A$ and B, compare lanes 2, 5, 8, and 11 with lanes 1, 4, 7, and 10). The presence of TF at a 2.5 -fold molar excess over ribosomes, which closely resembles the molar ratio found in vivo, reduced proteolysis of both constructs as demonstrated by the accumulation of the arrested nascent chains over time (Fig. 5A, lanes 3, 6, 9, and 12). Indirect chaperone effects of $\mathrm{TF}$, such as a putative stabilization of translation factors, can be excluded as the protective effect was not observed when the binding of TF to ribosomes was impaired (Fig. 5B, lanes 3, 6, 9, and 12). We conclude that TF provides a protected environment for nascent polypeptides during their ongoing synthesis.

Furthermore, we examined whether TF counteracts proteolysis when both TF and proteinase $\mathrm{K}$ are added after translation. Using monomeric and dimeric $\mathrm{m} 10$ as model substrates, no difference in the protection by co- or posttranslationally added TF was observed (Fig. 5C, compare lanes 6 and 8 and 10 and 12) indicating that TF productively re-associated with the ribosome-nascent chain complexes.

The DnaK System Cannot Efficiently Protect Nascent PolypeptidesThe DnaK chaperone compensates for the loss of TF activity in $\Delta$ tig cells and shows enhanced association with nascent polypeptide chains in the absence of TF $(11,13)$. This raises the question whether DnaK can directly substitute for TF in protecting nascent chains. To test the potential of the KJE system to protect nascent polypeptides, we supplemented our $\mathrm{t} / \mathrm{t}$ system, which contained reduced levels of DnaK (0.5 $\mu \mathrm{M})$ and lacked DnaJ, with $5 \mu \mathrm{M}$ DnaK, $1 \mu \mathrm{M}$ DnaJ, and $1 \mu \mathrm{M}$ GrpE. In separate samples we added DnaK and DnaJ but omitted the GrpE nucleotide exchange factor, which traps DnaK-substrate complexes and thereby should enhance a potential protective effect of DnaK. This KJ trap was functionally active in the $\mathrm{t} / \mathrm{t}$ system, since the addition of $\mathrm{KJ}$ completely prevented the folding of newly synthesized luciferase into its active state (data not shown).

In contrast to $\mathrm{TF}, \mathrm{KJE}$ and $\mathrm{KJ}$ did not provide protection of nascent m10 constructs (Fig. 6A). This failure was neither due to the lack of interaction between DnaK and $\mathrm{m} 10$ as demonstrated by cross-linking experiments nor to the inactivation of the KJE system by proteinase $\mathrm{K}$ (data not shown).

However, KJE and KJ protected nascent ICDH polypeptides of 11 and $19 \mathrm{kDa}$ to some extent, whereby the KJ trap was slightly more efficient (Fig. 6B, e.g. compare lanes 19 and 20). This protective effect was dependent on the length of the arrested ICDH polypeptide, with the best protection by KJE observed for the shortest ( $11 \mathrm{kDa}$ ) ICDH construct tested (Fig. 6B, lanes 27 and 28). Importantly, in all cases tested, TF displayed over the entire time period analyzed a significantly higher capacity to protect nascent chains than KJE. Together these data indicate that KJE cannot fully substitute TF in shielding nascent polypeptides.

\section{DISCUSSION}

In this study, we analyzed the potential of TF to shield nascent polypeptides exposed at the ribosome by performing protease protection assays. To this end we synthesized nascent polypeptide chains differing in origin, size, and folding status using an in vitro transcription/ translation system and tested their susceptibility to proteinase K digest.


FIGURE 5. TF protects arrested nascent polypeptides co- and posttranslationally. $A$ and $B$, synthesis of $\left[{ }^{35}\right.$ S $]$ methionine-labeled arrested nascent polypeptides was performed using either wt ribosomes in the presence of $1 \mu \mathrm{M} \mathrm{BSA}$ or $1 \mu \mathrm{M}$ wt TF $(A)$ or L23 mutant ribosomes (VSE/AAA) in the presence of $1 \mu \mathrm{M}$ BSA or $1 \mu \mathrm{m}$ TF-FRK/AAA (TF*) ribosome-binding mutant $(B)$. In contrast to all other experiments, PK was added during ongoing translation of nascent $2 \mathrm{~m} 10$ or $19-\mathrm{kDa}$ ICDH where denoted. At the indicated time points after PK addition, translation and PK digest were stopped simultaneously, and the remaining arrested polypeptides were isolated. Full-length products are shown. $C$, BSA $(5 \mu \mathrm{M})$ and TF $(5 \mu \mathrm{M})$ were either added prior to translation (labeled with " $\left.c o^{\prime \prime}\right)$ of arrested $\mathrm{m} 10$ and $2 \mathrm{~m} 10$ constructs or, different to all other experiments, after translation preceding PK addition (marked with "post"). PK digests were carried out as described in the legend to Fig. 2; full-length products are depicted.

The set of stalled model substrates comprised a folded SH3 domain, random coil constructs exposing one to five m10 modules, different lengths of truncated ICDH chains (11-45 kDa) (Figs. 2-6), and 15-kDa constructs of pyruvate kinase and firefly luciferase (data not shown). While the folded SH3 domain remained proteinase K-resistant, unfolded nascent polypeptides were rapidly degraded upon proteinase K addition in our in vitro system lacking TF. In contrast, the presence of TF counteracted the proteolysis of all non-native arrested polypeptides tested. Consistent with the rapid mode of TF substrate binding and release, which is required for the efficient folding of proteins on the time scale of seconds up to minutes (29), the protection provided by TF was transient in all cases tested.

The impairment of the ribosome binding of TF resulted in the loss of nascent polypeptide protection. Moreover, TF was unable to retard degradation of newly synthesized polypeptides released from the ribosome. Thus, TF displayed its shielding function only in its ribosome-bound state and specifically for nascent chains still connected to the peptidyltransferase center. This finding can be explained by the low affinity of TF for substrates in solution. The localization of TF next to the ribosomal polypeptide exit tunnel allows TF to associate much more efficiently with ribosome-tethered substrates. This assumption is consistent with earlier data showing that the chaperone function of TF in vivo requires its association with ribosomes (4). Interestingly, we observed a similar degree of protection irrespective whether TF was added co- or posttranslationally, which was tested for random coil constructs exposing up to $15 \mathrm{kDa}$ outside the tunnel. This result indicates that TF can 
FIGURE 6. The KJE chaperone system cannot protect nascent polypeptides like TF. BSA ( 5 $\mu \mathrm{M}), \operatorname{TF}(5 \mu \mathrm{M})$, the entire KJE system (5 $\mu \mathrm{m}$ DnaK, 1 $\mu \mathrm{M}$ DnaJ, and $1 \mu \mathrm{M}$ GrpE), or the KJ trap (5 $\mu \mathrm{m} \mathrm{DnaK}$, $1 \mu \mathrm{m}$ DnaJ; see "Results" for details) were added prior to synthesis of $\mathrm{m} 10(A)$ and ICDH $(B)$ derived nascent polypeptides. PK assays were performed as described (see Fig. 2). Note that the amounts of synthesized constructs were slightly increased in the presence of KJE and $\mathrm{KJ}$ trap for all $\mathrm{m} 10 \mathrm{con}$ structs ( $A$, lanes 3 and 4,7 and 8 , and 11 and 12 ) and $11-\mathrm{kDa}$ ICDH ( $B$, lanes 3 and 4 ). Boxed areas highlight the residual full-length nascent chains after 2 or 4 min of PK digest. Two prominent degradation intermediates generated by PK are indicated by asterisks (B). Bands running $30 \mathrm{kDa}$ higher than the individual full-length products represent fulllength polypeptides attached to tRNA.


productively re-associate with ribosomes carrying exposed nascent polypeptides in the size of protein domains and suggests that cycles of ribosome binding and release of TF are possible during ongoing translation. Such a TF cycling could be particularly important during the synthesis of multidomain proteins, which may require a repetitive chaperone action of TF for their productive de novo folding.

Analysis of the NC protein revealed that this TF fragment was able to protect all non-native constructs tested and that the PPIase domain of TF provides only a minor contribution to the shielding activity of TF. For example, TF and NC similarly protected nascent polypeptides of ICDH $(19 \mathrm{kDa})$ and $\mathrm{m} 10(11 \mathrm{kDa})$ during proteinase K digestion. This is consistent with the observation that in vivo, the NC fragment substitutes for wt TF and efficiently prevents the aggregation of newly synthesized proteins $(19,30)$. In contrast, the KJE chaperone system, which can compensate the lack of TF in vivo $(11,13)$, could not protect nascent polypeptides to an extent comparable with TF. DnaK was not able to protect random coil m10 constructs or the 33-kDa ICDH construct and was clearly less efficient in protecting ICDH constructs of 11 and $19 \mathrm{kDa}$ even when DnaK was artificially trapped in a substrate-bound state. However, the KJE system efficiently supported the folding of newly synthesized nascent polypeptides to their native states in the $t / t$ system demonstrating the general functionality of this system under our assays conditions (data not shown). Thus, the shielding capability seems to be an intrinsic property of ribosome-bound TF and cannot be fully substituted by DnaK. In vivo, DnaK may compensate the loss of TF through its abilities to actively promote folding processes and to repair misfolded proteins (31).

Investigating $\mathrm{ICDH}$ as a cytosolic E. coli protein, which is known to depend on TF for its productive folding in vivo (12), we found that TF protected all stalled nascent ICDH variants from degradation including the largest construct of $45 \mathrm{kDa}$, which covered almost the entire ICDH sequence. It is difficult to predict whether such large ICDH polypeptides already display significant secondary or tertiary structure, which may contribute to the protective TF effect we observed. Therefore we investigated artificial random coil $\mathrm{m} 10$ constructs that do not form any secondary structure. Again, TF shielded all tested m10 constructs exposing from 7 up to $37 \mathrm{kDa}$ outside the ribosomal tunnel, whereby only in the case of the m10 monomer and dimer, which exposed up to $15 \mathrm{kDa}$ outside the ribosome, the majority of full-length product remained undigested during proteinase $\mathrm{K}$ treatment. Interestingly, reducing $\mathrm{TF}$ ribosome binding by utilizing mutant ribosomes (L23-VSE/AAA) in combination with wt TF, we observed differences between ICDH and m10 as model substrates. While TF still protected all ICDH constructs to some degree, TF was unable to counteract the degradation of the random coil $\mathrm{m} 10$ nascent chains (data not shown). These differences in protection may be explained by a stronger interaction of TF with its in vivo substrate ICDH compared with the artificial m10 construct.

Taken together, these data point out (i) that TF functions as a general protective shield for nascent polypeptides differing in size and folding status, wherein the degree of protection varies for different kinds of substrates and (ii) that the protection by TF is obviously not restricted to short stretches of nascent chains immediately adjacent to the tunnel exit but is evident for larger sized unfolded nascent polypeptides. How may TF accomplish its function as a protective shield? Two alternative mechanisms can be envisioned that are not mutually exclusive and may depend on the size and folding status of the particular nascent polypeptide. On one hand, TF may exert its protective function by binding to a small stretch within the unfolded nascent substrate, which is sufficient 
to transiently hinder the ability of the protease to attack the nascent substrate. On the other hand, based on our results, it is tempting to speculate that entire protein domains can be accommodated underneath the TF chaperone. This scenario would also permit the co-translational folding of nascent polypeptides in such a protected environment. However, our finding that TF was able to transiently shield nascent polypeptides exposing up to 37 or $41 \mathrm{kDa}$ outside the ribosome raises the question of how such large proteins can be accommodated underneath TF. It is possible that the cavity formed between the ribosomal surface and the $\mathrm{N}$-terminal tail and $\mathrm{C}$-terminal arms of TF can adopt a significantly larger volume $(>14 \mathrm{kDa})$ than originally anticipated based on the structural models $(5-7,15)$. Another explanation is that large nascent polypeptides are incorporated underneath the entire TF molecule additionally involving the $\mathrm{C}$-terminal region beyond the $\mathrm{C}$-terminal arms and perhaps the PPIase domain. The latter assumption is supported by our observation that the slight contribution of the PPIase domain to the shielding effect of TF was more pronounced for $\mathrm{m} 10$ constructs exposing more than $15 \mathrm{kDa}$ outside the ribosomal tunnel. By means of our protease protection assays, we are not yet able to discriminate between these alternative scenarios of how TF may function as a protective shield on the molecular level, and further experimental efforts will be required to clarify the detailed mechanism of TF action.

Acknowledgments-We are thankful to members of the Deuerling, Mayer, and Bukau laboratories, in particular R. D. Wegrzyn, M. P. Mayer, and A. Mogkfor comments on the manuscript and R. Zahn for the donation of purified DnaK. We thank L. Serrano, C. Schaffitzel, and N. Ban for their generous gift of plasmids.

\section{REFERENCES}

1. Young, J. C., Agashe, V. R., Siegers, K., and Hartl, F. U. (2004) Nat. Rev. Mol. Cell. Biol. 5, 781-791

2. Bukau, B., Deuerling, E., Pfund, C., and Craig, E. A. (2000) Cell 101, 119-122

3. Frydman, J. (2001) Annu. Rev. Biochem. 70, 603-647

4. Kramer, G., Rauch, T., Rist, W., Vorderwülbecke, S., Patzelt, H., Schulze-Specking, A., Ban, N., Deuerling, E., and Bukau, B. (2002) Nature 419, 171-174.

5. Ferbitz, L., Maier, T., Patzelt, H., Bukau, B., Deuerling, E., and Ban, N. (2004) Nature 431, $590-596$
6. Baram, D., Pyetan, E., Sittner, A., Auerbach-Nevo, T., Bashan, A., and Yonath, A. (2005) Proc. Natl. Acad. Sci. U. S. A. 102, 12017-12022

7. Schlunzen, F., Wilson, D. N., Tian, P., Harms, J. M., McInnes, S. J., Hansen, H. A., Albrecht, R., Buerger, J., Wilbanks, S. M., and Fucini, P. (2005) Structure (Camb.) 13, 1685-1694

8. Valent, Q. A., Kendall, D. A., High, S., Kusters, R., Oudega, B., and Luirink, J. (1995) EMBO J. 14, 5494-5505

9. Hesterkamp, T., Hauser, S., Lütcke, H., and Bukau, B. (1996) Proc. Natl. Acad. Sci. U. S. A. 93, 4437-444.1

10. Hartl, F. U., and Hayer-Hartl, M. (2002) Science 295, 1852-1858

11. Deuerling, E., Schulze-Specking, A., Tomoyasu, T., Mogk, A., and Bukau, B. (1999) Nature 400, 693-696

12. Deuerling, E., Patzelt, H., Vorderwülbecke, S., Rauch, T., Kramer, G., Schaffitzel, E., Mogk, A., Schulze-Specking, A., Langen, H., and Bukau, B. (2003) Mol. Microbiol. 47, 1317-1328

13. Teter, S. A., Houry, W. A., Ang, D., Tradler, T., Rockabrand, D., Fischer, G., Blum, P., Georgopoulos, C., and Hartl, F. U. (1999) Cell 97, 755-765

14. Ludlam, A. V., Moore, B. A., and Xu, Z. (2004) Proc. Natl. Acad. Sci. U. S. A. 101, $13436-13441$

15. Baram, D., and Yonath, A. (2005) FEBS Lett. 579, 948 -954

16. Stoller, G., Ruecknagel, K. P., Nierhaus, K. H., Schmid, F. X., Fischer, G., and Rahfeld, J.-U. (1995) EMBO J. 14, 4939-4948

17. Blanco, F. J., Angrand, I., and Serrano, L. (1999) J. Mol. Biol. 285, 741-753

18. Mitra, K., Schaffitzel, C., Shaikh, T., Tama, F., Jenni, S., Brooks, C. L., III, Ban, N., and Frank, J. (2005) Nature 438, 318-324

19. Kramer, G., Rutkowska, A., Wegrzyn, R. D., Patzelt, H., Kurz, T. A., Merz, F., Rauch, T., Vorderwülbecke, S., Deuerling, E., and Bukau, B. (2004) J. Bacteriol. 186, 3777-3784

20. Hesterkamp, T., Deuerling, E., and Bukau, B. (1997) J. Biol. Chem. 272, 21865-21871

21. Buchberger, A., Valencia, A., McMacken, R., Sander, C., and Bukau, B. (1994) EMBO J. 13, 1687-1695

22. Schönfeld, H.-J., Schmidt, D., Schröder, H., and Bukau, B. (1995) J. Biol. Chem. 270, 2183-2189

23. Bukau, B., and Walker, G. C. (1989) J. Bacteriol. 171, $6030-6038$

24. Muller, M., and Blobel, G. (1986) Curr. Top. Microbiol. Immunol. 125, 33-41

25. Stoddard, B. L., and Koshland, D. E., Jr. (1993) Biochemistry 32, 9317-9322

26. Musacchio, A., Noble, M., Pauptit, R., Wierenga, R., and Saraste, M. (1992) Nature 359, 851-855

27. Evans, M. S., Ugrinov, K. G., Frese, M. A., and Clark, P. L. (2005) Nat. Methods 2, $757-762$

28. Nakatogawa, H., and Ito, K. (2002) Cell 108, 629-636

29. Maier, R., Scholz, C., and Schmid, F. X. (2001) J. Mol. Biol. 314, 1181-1190

30. Genevaux, P., Keppel, F., Schwager, F., Langendijk-Genevaux, P. S., Hartl, F. U., and Georgopoulos, C. (2004) EMBO Rep. 5, 195-200

31. Bukau, B., and Horwich, A. L. (1998) Cell 92, 351-366 